REDES- Revista hispana para el análisis de redes sociales.

Vol.3,\#1, sept-nov. 2002.

http: // revista-redes.rediris.es

\title{
Presentación: Tendiendo puentes, de Lilnet a Redes
}

\author{
Ainhoa de Federico ${ }^{1}$ \\ Universidad de Lille (Francia) \\ Universidad Pública de Navarra (España)
}

“El análisis de redes sociales ofrece un medio

para establecer el lazo entre las explicaciones a

nivel micro y a nivel macro"2

(Knoke \& Kuklinski, 1982: 13)

Este volumen de Redes recoge una selección ${ }^{3}$ de ponencias -traducidas- de entre las más de cuarenta presentadas en la III Conferencia Temática Europea de Analistas de Redes Sociales "LILNET. Relaciones entre micro y macro: contribuciones del análisis estructural" celebrada, con el apoyo de la INSNA ${ }^{4}$, en Lille (Francia) del 30 al 31 de Mayo 2002. La organización de esta Conferencia Temática Europea pretendió continuar la dirección apuntada por las dos Conferencias Temáticas precedentes celebradas en Groningen (1992) sobre "Muestreo mediante bola de nieve y metodologías ascendentes asociadas" y en Maastricht (1999) sobre "Muestreo de Redes".

La existencia de estas Conferencias Temáticas, al igual que las Conferencias Europeas desde $1989^{5}$, ahora oficialmente integradas cada tres años en las SUNBELT ${ }^{6}$, son una muestra más de la buena salud del análisis de redes sociales. Efectivamente, el interés por la aproximación estructural se ve acrecentado y ampliado fuera del ámbito norteamericano, lo que permite la inclusión de nuevas ideas y perspectivas. La creación de la revista REDES y la sinergia creciente del análisis de estructural en español son también muestras de esta fantástica expansión y enriquecimiento del análisis de redes sociales en el mundo. Nada mejor que combinar los impulsos y permitir a una iniciativa europea alcanzar al ámbito hispanohablante.

1 Enviar correspondencia a: Ainhoa de Federico, federico@univ-lillel.fr

2 "Network analysis offers a mean for bridging the gap between macro- and micro-level explanations"

3 La totalidad de los resúmenes de la conferencia están disponibles, ordenados por sesión, en http://www.univ-lillel.fr/lilnet/program/proe.htm; para obtener los textos originales en inglés y francés, o tener acceso a los textos no publicados en el presente número, los lectores pueden dirigirse directamente a los autores.

4 La INSNA es la asociación profesional internacional de analistas de redes sociales. Para más información ver http://www2.heinz.cmu.edu/project/INSNA/ o su nuevo web-site http://www.sfu.ca/ insna/

${ }^{5}$ Las Conferencias Europeas han sido: en Groningen (1989), París (1991), Colonia (1993?), Londres (1995), Sitges (1998), Budapest (2001) y la próxima tendrá lugar en Eslovenia (2004).

6 Las conferencias SUNBELT son las conferencias internacionales de análisis de redes sociales organizadas por la INSNA. Primero organizadas alternando la costa este/oeste de Norte América, ahora alternando la costa este/oeste de América con Europa. 
La cuestión de la división entre los niveles micro y macro y su relación es considerada por algunos autores, como Collins (1988) como una de las cuestiones clave en la sociología contemporánea. De acuerdo con él, el análisis de redes sociales y las teorías estructurales son prometedoras para establecer el puente entre ambos niveles. Tradicionalmente se podría decir que la manera en que las ciencias sociales han formulado la relación micro-macro han reflejado diversos postulados teóricos: los mecanismos de mercado, las creencias religiosas respecto a "lo sagrado", o la política formal han sido consideradas centrales para los órdenes sociales multinivel. Sin embargo, el problema de la relación micro-macro, no es un problema consustancial a cualquier aproximación teórica sobre la realidad social. Este problema surge o se plantea dentro de un marco teórico específico y de una concepción concreta de las ciencias sociales. La cuestión de la división entre los niveles micro y macro, y su relación, es una consecuencia directa e inmediata de esta concepción, por lo tanto es necesario hacer referencia a ella.

Para abordar este cometido, tomemos como punto de partida el marco de formulación general propuesto por Coleman (1990) en el primer capítulo de su obra monumental Foundations of Social Theory. Esta formulación presenta la ventaja de ser conocida y ampliamente aceptado por los analistas de redes sociales ${ }^{7}$. En esta aproximación, se considera que el problema central de las ciencias sociales es explicar el funcionamiento de diferentes sistemas sociales. Sin embargo, dado que a veces el número de sistemas es limitado, o que éstos no son observables directamente en tanto que sistemas, la manera de aproximarse al estudio de los sistemas es mediante el examen y la observación de los procesos internos al sistema, esto es del funcionamiento e interacción de las diversas partes del sistema. A priori, la identificación del sistema y de sus partes depende del problema considerado, sin embargo esta aproximación identifica la unidad mínima inferior natural en las ciencias sociales con el individuo y su comportamiento. Aunque el objetivo final no es la explicación del comportamiento individual ${ }^{8}$, ésta aproximación considera que explicarlo o comprenderlo de manera suficiente es un paso intermedio y necesario para la explicación del funcionamiento de los sistemas, ya que se considera que el comportamiento del sistema es la resultante de las acciones de sus partes componentes. Dada la identificación de la unidad "natural" de las ciencias sociales con las unidades de seres humanos, sin que por ello el interés final sea explicar su comportamiento, a esta aproximación se le llama individualismo metodológico.

Evidentemente existen grandes discrepancias sobre qué se considera una explicación suficiente del comportamiento de los individuos y en el contenido de éstas. En cuanto al contenido, la más evidente es sin duda la diferencia entre las aproximaciones tradicionales de las ciencias económicas y la sociología, concepciones de los individuos radicalmente opuestas. Siguiendo el resumen propuesto por Lindenberg (1990), mientras que la economía ha postulado tradicionalmente un homo oeconomicus perfectamente informado, egoísta, aislado socialmente y maximizador-consumidor, la sociología tradicional por su parte ha propuesto una visión del homo sociologicus socializado, adoptador de roles y conformador a las reglas, o al menos buscador de reconocimiento social. Existen actualmente intentos de conciliar o volver complementarias las aproximaciones

7 Aunque no por todos, Pizarro (2000, ver también en éste número), presenta una interesante y feroz crítica al individualismo metodológico y en particular a la exposición de Coleman, la perspectiva radicalmente diferente de Pizarro presenta, por lo tanto, implicaciones distintas en la relación micromacro.

8 Lindenberg discrepa con Coleman en que la atención final, el objetivo final de las ciencias sociales sea la explicación de sistemas por dos motivos, el primero, moral, porque, finalmente, los sistemas sociales nos interesan en tanto que tienen un impacto sobre el bienestar, felicidad, condiciones de existencia de los individuos, el segundo, empírico, porque una gran parte de las investigaciones no portan sobre sistemas: funcionamiento de instituciones, normas, proceso de globalización...; sino sobre el nivel micro colectivo o agregado: tasas de suicidio, de éxito escolar... 
económica y sociológica con la propuesta de un modelo de individuo que Lindenberg llama homo socio-oeconomicus.

En cuanto a la amplitud de la concepción sobre los individuos, a la que se considera suficiente, también existen discrepancias. No es necesario abordar las diferencias disciplinares obvias, por ejemplo, entre sociología y psicología, que acuerdan un interés de profundidad bien diferente a la explicación del comportamiento individual, para encontrar grandes diferencias. Incluso dentro del individualismo metodológico en sociología encontramos diferencias importantes; por ejemplo, entre la concepción más "delgada" de las "buenas razones" de Boudon (1981) y la concepción compleja y amplia del homo socio-oeconomicus de Lindenberg (2002). En todo caso, ya sean de un tipo o de otro, las concepciones ligadas al individualismo metodológico tratan de actores sociales. La cuestión de la relación entre macro y micro no siempre requiere descender al nivel de los individuos como nivel micro - aunque este sería su límite inferior- pero en cualquier caso requiere considerar que en el nivel micro nos encontramos con actores sociales ya sean individuales o colectivos.

Volviendo a Coleman para tratar de situar mejor la cuestión, ahora que tenemos una concepción del nivel micro, las cuestiones sobre la relación entre macro-micromacro tratan entonces sobre la explicación de fenómenos al nivel macro, es decir al nivel del sistema que nos interesa comprender o explicar, basándose en teorías sobre el comportamiento de los actores al nivel menor, que están comprendidos en el nivel macro del sistema, y teniendo en cuenta el contexto que éste representa. Tal como lo expresa el esquema (Figura 1), el nivel macro es un sistema en transición. El nivel macro impone limitaciones y oportunidades a los actores. Estos, en el nivel micro, dadas las condiciones y su capacidad de actuar, desarrollan comportamientos que a su vez, de un modo u otro, inciden en la transformación del sistema macro.

Nivel Macro

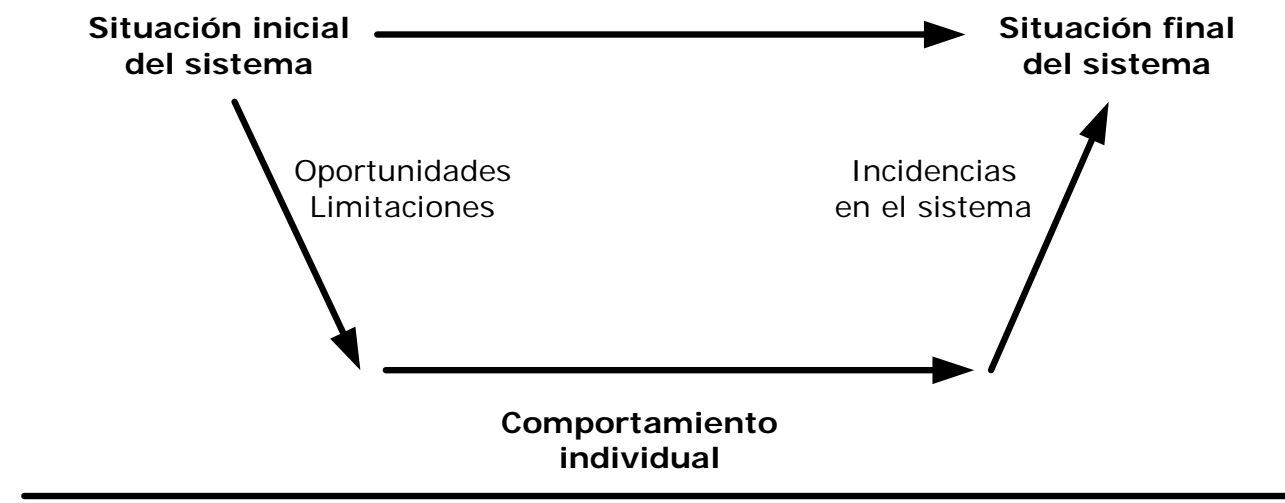

Nivel Micro

Este esquema general de Coleman permite una definición abierta del "nivel macro" y del "nivel micro". De hecho parece una definición a priori puramente contextual y, como veremos en las contribuciones a este volumen, dentro de este marco abierto, autores diferentes han tomado definiciones o "localizaciones" diferentes de los niveles macro y micro, por ejemplo Snijders toma como nivel macro una red personal total - al fin y al cabo un sistema que dentro de la tradición sociológica se puede considerar bastante micro- y como nivel micro a los individuos, mientras que Coleman mismo ofrece ejemplos que ascienden a un nivel mucho mayor, como el sistema capitalista - considerados también macro por la tradición sociológica. De manera consistente, de algún modo, con la tradición sociológica sería una osadía 
llamar sistema macro a las redes personales, incluso pudiendo ser estas ilimitadas y atravesar la totalidad de la sociedad -incluso el planeta-, para ello se propone a menudo el término meso (Ferrand y Lazega en este número, aunque no son ni los únicos ni los primeros).

Esto nos lleva a considerar que además de la aproximación contextual a la cuestión de la relación micro-macro, al menos de forma implícita, algunos autores también parecen considerar importante una distinción sustantiva entre estos niveles. Mientras que en la aproximación contextual es suficiente identificar un sistema $X$ compuesto por actores $Y$, independientemente del nivel de institucionalización e independientemente de la distancia a los individuos humanos de $X$ e $Y$; en una aproximación sustantiva sería necesario definir explícitamente cual es el nivel micro qué tipos de sistemas de agencia intermedia informal constituyen un posible nivel meso y a partir de qué nivel de institucionalización se puede considerar que hablamos del nivel macro. De forma provisional, concordando con la tradición sociológica es posible proponer definir como nivel macro a los sistemas sociales institucionalizados, aquellos objetos de estudio tradicionales de la sociología, como nivel micro a los actores y su comportamiento individual ${ }^{9}$ y como nivel meso a las redes de actores.

El esquema propuesto por Burt (1982) ya contiene los tres niveles de que hablamos, aunque no utilicen exactamente el mismo vocabulario. Lo presentamos aquí ligeramente adaptado.
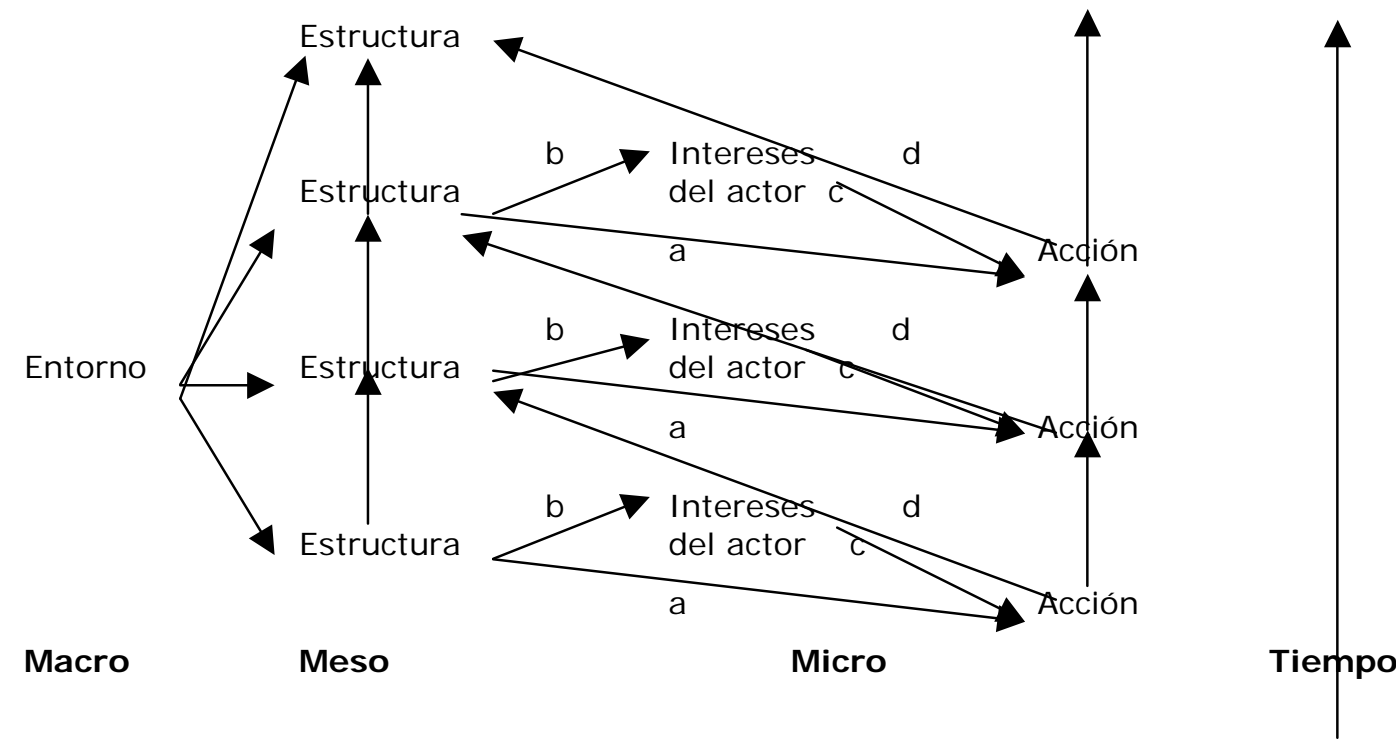

Degenne y Forsé (1994) expresan las relaciones entre los tres niveles de la manera siguiente: a) la estructura pesa formalmente sobre la acción según un determinismo débil [...]. b) La estructura afecta la percepción de los intereses de los actores [...] c) el principio de racionalidad : si el individuo es racional, establece una elección conforme a sus intereses ( $u$ orden de preferencias)[...]. d) La estructura es el efecto emergente de las interacciones [...]. No hay que olvidar que esta estructura está sumergida en un entorno más amplio [...]. En este esquema encontramos ya una especificación de las relaciones entre dos niveles: el nivel que

9 Pizarro discreparía recordando que la primera institución jurídica es precisamente la de persona en el Derecho Romano de Tertuliano y que "Ios procesos de atribución de nombre propio están asociados con los sistemas de parentesco y otras estructuras sociales e instituciones" $(2000,171)$. 
aquí llamamos micro - individual, siguiendo las ideas del individualismo metodológico y del principio de racionalidad de los actores, y el nivel que aquí llamamos meso, es decir la estructura de posiciones más o menos informal emergente de las interacciones de los individuos, y que por su parte modifica la definición de la situación de los actores, que habitualmente llamamos redes sociales. Sin embargo la relación entre el nivel meso y el nivel macro está conceptualizada en este esquema en una sola dirección: la de que el entorno social más amplio impone un contexto que implica limitaciones de un tipo u otro a la red. Efectivamente, parece más evidente que el contexto institucional pueda ejercer restricciones, prescripciones y proscripciones sobre lo que ocurre en su interior. Sin embargo falta el camino teórico de vuelta entre el nivel meso y el nivel macro. En efecto, si bien parece difícil que un individuo cualquiera pueda ser capaz de incidir en el sistema de regulación del nivel macro, individuos en posiciones particulares de la estructura o coaliciones de individuos en estructuras particulares pueden perfectamente ejercer estas influencias, como lo sugieren diversas teorías sobre la regulación (ver Lazega en este número), o el estudio de las elites (ver Alcántara y Casasola, y Adler-Lomnitz, en este número), entre otros. Un esquema general abierto sobre las relaciones entre macro, meso y micro se podría representar así:

\section{Nivel Macro, institucional}

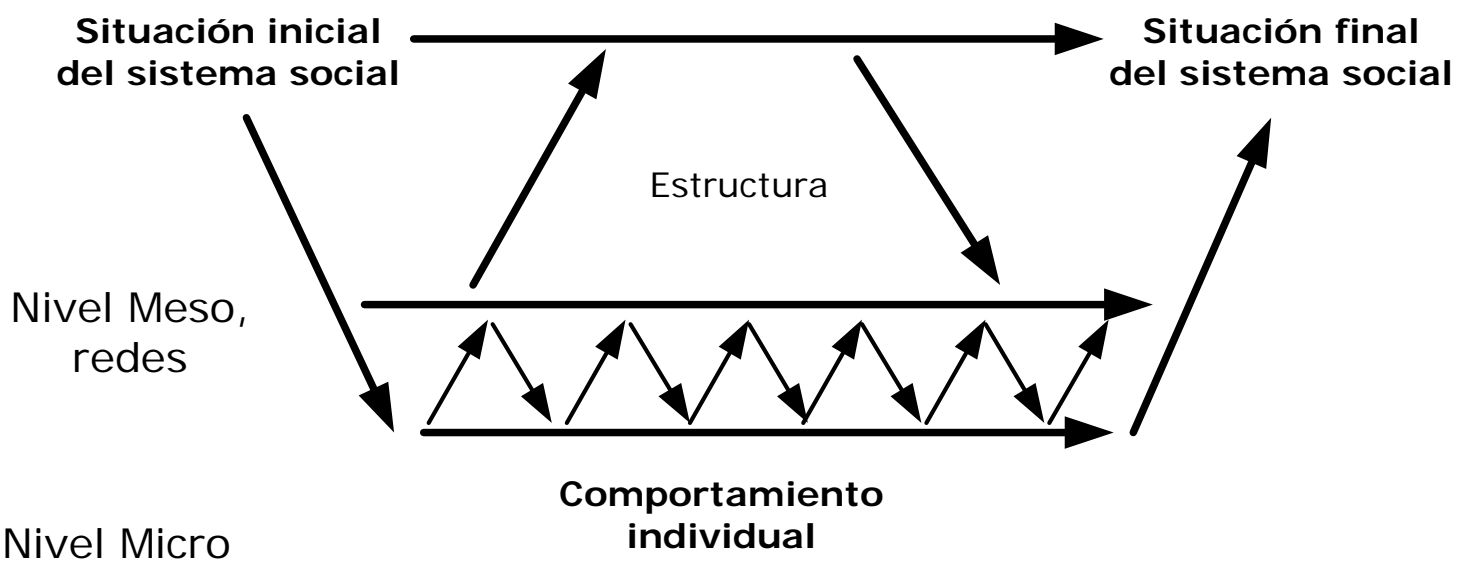

A partir de este marco es posible conceptualizar transiciones múltiples. Como ya aparece en el esquema de Burt, la introducción de un nivel meso estructural, que constituye el entorno inmediato de definición de la situación, de control y de acceso diversos recursos sociales (estatus y todo tipo de formas de capital) de los actores, tiene incidencia en la reformulación de los objetivos por parte de los actores, y por lo tanto tiene consecuencias en el nivel micro. A su vez, las acciones de los individuos inciden en la estructura meso. Pero también la capacidad de los actores de politizar sus intercambios (ver Lazega en este número) y tratar de participar en los procesos de regulación y redefinición de las reglas del sistema está mediada por las posiciones sociales de éstos en la estructura, por lo tanto el nivel meso también influye en el nivel macro, el cual a su vez impone las reglas de funcionamiento general permitiendo, facilitando, dificultando tal o cual estructura. Así la aproximación estructural añade capacidad explicativa mediante la posibilidad del estudio dinámico de las interrelaciones entre los niveles macro, meso y micro y por lo tanto permite establecer de modo más preciso la relación entre micro y macro.

Por otra parte, es necesario recordar que, además de ser capaz de ofrecer proposiciones teóricas capaces de dar cuenta de la relación dinámica entre micro, meso y macro, la aproximación estructural se caracteriza también por el desarrollo de métodos sofisticados de análisis de redes sociales que permiten implementar de manera precisa las diferentes hipótesis estructurales. Hoy en día se dibujan nuevas 
tendencias en el análisis de redes sociales que podrían converger hacia el objetivo de modelar la relación micro-meso, y meso-macro. Por ejemplo, en uno de los extremos, los analistas de redes contemporáneos han sido formados (en particular a causa del modelado de bloques 'block modeling') a conceptualizar la estructura en términos de posiciones sociales y de interdependencias entre ellas (en este número la original propuesta de Pizarro). En esta aproximación se examina la manera en que los individuos de una red se distinguen a partir de sus perfiles relacionales. $O$ como lo sugiere Pizarro, la definición de lugares y redes de lugares más allá de los individuos particulares que los ocupan. En este caso la dinámica concierne la emergencia - eventual- de lugares diferentes y por lo tanto la reorganización del conjunto de lugares y su red de relaciones.

En el otro extremo, los modelos estadísticos actuales como p2, p*, SIENA (ver Snijders en este volumen) se centran en los procesos locales que serían responsables de la estructura de conjunto. Estos presuponen limitaciones subestructurales locales y afirman que cuando el análisis ha tenido en cuenta estas tendencias locales sub-estructurales, la estructura de conjunto se establece de alguna manera "independientemente" de estos elementos, en el sentido de que no habría estructura global más allá de las tendencias sub-estructurales locales. La estructura global y las tendencias estructurales (como por ejemplo la bipartición de una red en cliques de amigos) pueden ser explicadas por limitaciones locales (por ejemplo la tendencia de los amigos de mis amigos a convertirse en mis amigos). Como lo muestra el artículo de Pattison (en este volumen) existen intentos de conciliar o al menos comparar ambas aproximaciones.

Esta introducción ha pretendido situar la cuestión de la relación micro-macro en su marco teórico, al mismo tiempo que ofrecer ejemplos de aproximaciones desarrolladas actualmente dentro del análisis estructural para explicar esta relación. Más que tratar de establecer una teoría definitiva, capaz de cubrir todas las propuestas teóricas, a veces de índole bien diferente, la intención ha sido ofrecer una marco que permita situar el tipo de problemas y perspectivas abordados en este número. Creo que la distinción entre los niveles macro, meso y micro y el reconocimiento de las interacciones recíprocas entre ellos permite situar mejor una mayor cantidad de las contribuciones a este número. Este era mi objetivo, establecer un marco de presentación de éstos. Para deleitarse con las diferentes propuestas teóricas, heurísticas y técnicas, baste remitir a los autores de este número, en su gran mayoría grandes figuras del análisis de redes sociales contemporáneo.

A continuación se esbozan brevemente las contribuciones que están previstas para este volumen de Redes. Dado que son muchas, se procederá a su publicación electrónica por entregas, a medida que estén disponibles las traducciones de los textos. Por ahora, esperamos que esta presentación sirva para proporcionar una visión de conjunto de las contribuciones realizadas por los diferentes autores.

\section{¿Qué es el nivel meso? Una perspectiva neo estructural sobre la política. Emmanuel Lazega}

Este artículo se centra en la relación entre los niveles micro y macro de análisis asumiendo de entrada que los actores tienden a politizar sus intercambios y la gestión de sus recursos. Por ejemplo, estos actores intentan tanto como pueden modelar y remodelar sus estructuras de oportunidades principalmente mediante la creación y mantenimiento de nichos sociales y compitiendo por diversas formas de estatus. Tal proposición general conduce a una teoría concebida en un sentido amplio de la racionalidad y la estructura que incluye la capacidad de los actores de contextualizar su acción. En mi opinión esta perspectiva ofrece una aproximación neo-estructural a la política - la política, como la mayor conexión entre el nivel micro y el nivel macro. En esta perspectiva, tanto la política formal representativa como los usos informales de las interdependencias de recursos se combinan para 
dar cuenta de los procesos principales mediante los que los colectivos - como comunidades políticas grandes o pequeñas- son dirigidos por los individuos que restringen en sus esfuerzos por mantener la solidaridad el control y la regulación. Esta perspectiva trata de combinar las ideas de la elección racional con perspectivas interaccionistas más estratégicas y simbólicas - pero también con la formalización y el modelado sistemático ofrecido por los estadísticos contemporáneos. Los ejemplos presentados provienen principalmente del área de la sociología económica. Examino las relaciones entre estos conceptos examinando el comportamiento de actores interdependientes y los mecanismos sociales de su cooperación.

\section{Redes sociales y partidos políticos en Chile}

Larissa Adler-Lomnitz

En este estudio se describe el origen y la evolución de dos partidos políticos chilenos (el Partido Radical y el Partido Demócrata Cristiano) por medio del análisis de las redes sociales que los originaron y que los componen. Se propone un modelo teórico que consiste en la combinación de un análisis estructural basado en el estudio de redes sociales y en la descripción del sistema simbólico que las retroalimenta. La estructura y función de las redes sociales depende de la dirección en la que se dan los intercambios - redes horizontales y redes verticales-, de lo que se intercambia, y de la articulación que se da entre las redes. En toda sociedad se dan intercambios simétricos y asimétricos, que van conformando redes horizontales y verticales. Estas redes se van articulando entre sí, conformando el tejido social. El predominio de unas sobre otras y su combinación dan el carácter a la cultura política (ejemplo, autoritaria vs. igualitaria). En el caso de Chile encontramos que se trata de una sociedad horizontalmente estructurada en clases sociales, al interior de las cuales se advierten redes sociales informales que eventualmente y por razones ideológicas se van formalizando en partidos políticos. Estos grupos igualitarios de amigos ejercen informalmente control sobre sus propios miembros creando barreras invisibles que los distinguen de los demás, y aunque si bien es cierto que en ellos surgen líderes "naturales", su liderazgo es condicional lo que permite el surgimiento de tendencias y facciones que a su vez pueden llegar a constituirse en nuevos partidos. Por ende, el sistema depende de negociaciones horizontales permanentes mediadas por un fuerte presidencialismo dentro de un sistema parlamentario fuerte y apoyado por una legitimidad casi fanática.

\section{El núcleo de la red de matrimonios en la elite de la Guatemala colonial} Narda Alcántara Valverde, Silvia Casasola Vargas y Douglas White

Siguiendo la definición de Houseman y White del núcleo de una red de matrimonios, los autores identifican el núcleo de la red de la élite de la Guatemala Colonial en el periodo de 1640 a 1820 en términos estructurales en relación con los conceptos de re-ligazón mediante matrimonio y de consolidación de la riqueza a través de la endogamia estructural. Comprobamos las hipótesis de la relación entre la estructura y la re-ligazón por matrimonio y la consolidación de la riqueza y la prominencia en una muestra de familias de la élite a partir de la base de datos creada por Casasola. La definición de Houseman y White es útil para explicar la estructura y la dinámica de los grupos cognaticios de descendencia, tales como el sistema de parentesco español. También proponen un método para estudiar el núcleo de prestigio de una red de matrimonios. La relevancia de este estudio reside en su capacidad de explicar la dinámica y la composición de una red de elite del nivel macro al micro y viceversa.

\section{Las comunidades locales como estructuras meso}

Alexis Ferrand

Entre el nivel micro y el nivel macro: meso. Si el nivel macro está definido por la regulación nacional, estatal o del mercado y el nivel micro por la regulación 
interpersonal, una agencia de regulación intermediaria de mayor importancia ha sido definida como "comunidad local" (Wellman, Fischer). Aparte de las dimensiones organizacionales (organizaciones locales, clubes, tiendas...) e institucionales (poder local, partidos...) locales, es posible definir algunas propiedades estructurales pertinentes de los sistemas de relaciones interpersonales privadas: uno es el grado de "cerrado" (closure) local (Barnes: redes locales vs. transversales), o la "dualidad" de estos sistemas. Apoyándose en la idea de que la eficiencia de los "puentes" reside en el tipo de cliques que conectan (Lin), es posible definir tipos variados de micro-estructuras, cuyas distribuciones en diferentes localidades son indicadores sucedáneos o aproximaciones de las diferentes meso estructuras de sistemas relacionales como combinaciones de redes "locales" y "no locales".

\section{Cadenas migratorias y redes personales: relaciones micro-macro} Isidro Maya Jariego y Luis Rull Muñoz

En las comunidades de inmigrantes, la variación de la composición de las redes y la estructura puede afectar las provisiones de apoyo social a través de esos lazos. Por lo tanto exploramos cómo el carácter de apoyo de la red personal (nivel individual de análisis) es condicionado por el proceso de migración de cadena (nivel de análisis de la red). Primero, proponemos una tipología de lazos fuertes, y analizamos si el carácter de apoyo es influido por su posición en la cadena migratoria. A continuación, proponemos una tipología de redes de apoyo social y analizamos si está relacionada con la cadena migratoria. Ambos análisis tienen en cuenta el momento de llegada y muestran las diferencias entre la primera generación y la segunda generación de inmigrantes. Discutimos los beneficios de un análisis que integre individuos y redes.

Amistad e identificación: las micro fundaciones de las pertenencias macro. Amigos europeos e identidad europea

Ainhoa de Federico

La idea inicial de esta presentación es que la pertenencia macro a "comunidades imaginadas" (Anderson 1983), tales como las naciones, están fundadas en la transferencia metonímica generalizada, fomentada por las instituciones, a los habitantes de cierto territorio, de la experiencia interpersonal de confianza y solidaridad.

El contrato social nacional está ideológicamente fundado en el modelo ideal de la solidaridad en las relaciones de amistad. El ejemplo más explícito de ello es el lema francés "liberté, égalité, fraternité". Esto no quiere decir que exista una correspondencia exacta entre estos dos tipos de relación, tal como Eisenstadt (1984) señala, la fraternidad ideal anunciada por la nación sirve para ocultar y justificar el poder y la instrumentalidad que el orden de la nación-estado ejerce sobre sus miembros. Al mismo tiempo, la amistad (en su acepción más amplia) sostiene el contrato social al mismo tiempo que debe ser relegada a la esfera privada debido a su potencial subversivo (Paine 1969).

Las relaciones de amistad a menudo ocurren dentro de la esfera de los estadosnación, puesto que sus instituciones estructuran la interacción y las interdependencias entre los actores a partir de la organización de la división social del trabajo. Sin embargo, ¿qué sucede con las relaciones trans-nacionales? La figura paradójica del "amigo extranjero" introduce una tensión entre los fundamentos de la solidaridad abstracta y la experiencia de la solidaridad interpersonal. ¿Queda esta tensión relegada a ser resuelta en la esfera privada o acaso tiene una incidencia en las pertenencias públicas macro sociales reclamadas y proclamadas por los actores? 
Por añadido, en Europa existe un contexto institucional que permite y que intenta promover la identificación con Europa. ¿Podrían tales amistades europeas dar un contenido al envoltorio legal macro de la ciudadanía Europea, por otra parte vacío en términos de apego emocional, fomentando la identidad Europea?

Examinamos el efecto de relaciones de amistad trans-nacionales en el caso de estudiantes Europeos participantes en programas Europeos de intercambio, sobre la percepción y categorización mutua en tanto que extranjeros y respecto a su identidad Europea.

\section{Redes y normas: integración micro y macro en comunidades en línea en Internet Christoph Müller}

La mayoría de las comunidades en línea en Internet como los Chats o Newsgroups están débilmente estructurados especialmente debido a la falta de reglas escritas y de pertenencia formal. La facilidad de las opciones de entrada y salida conducen a grandes cantidades de fluctuaciones y cambio de miembros. Además, las posibilidades de sancionar positivamente o negativamente el comportamiento son restringidas. Por lo tanto muchos Chats o Newsgroups son inestables, y se encuentran con serios problemas para establecer y mantener un orden social. ¿Cómo organizan las comunidades en línea su estructura social? ¿Hasta qué punto se pueden considerar los Chats o Newsgroups como "comunidades" o " grupos"?

Un estudio de diferentes servicios de comunidades en línea en Suiza muestra la importancia de ambos niveles, micro y macro, de "construcción comunitaria". La parte empírica de la investigación se basa en dos aproximaciones metodológicas: (a) una encuesta cuantitativa con entrevistas cara a cara revelando las redes personales de 101 usuarios frecuentes de Chats y Newsgroups, y (b) un análisis cualitativo del discurso que estudia el comportamiento en línea de los participantes de un Chat y un Newsgroup.

Los resultados de la investigación muestran cómo diferentes (micro-) actores están relacionados entre sí por relaciones de red, formando agrupamientos y subgrupos, mientras que la (macro-) estructura de las comunidades en línea es mantenida por normas técnicas y sociales. Estas normas incluyen el lenguaje, instituciones y contexto tecnológico. Ambos redes y normas son partes esenciales para la construcción de comunidades en línea: en un nivel micro, las relaciones sociales son mantenidas por la interacción personal, mientras que en un nivel macro están basadas en normas comunes.

\section{Macro-micro-macro y modelos estadísticos para redes}

Tom Snijders

Para llevar a cabo el trabajo empírico sobre temas macro-micro-macro, es indispensable tener modelos estadísticos que reflejen de manera plausible la estructura de los datos y las relaciones teóricas en la base de tales cuestiones. Una familia básica de modelos para los efectos macro-micro es ofrecida por el análisis multinivel. Una manera importante de estudiar las transiciones micro-macro es mediante simulación.

Los modelos sobre redes proponen otra perspectiva, particularmente apropiada, a los temas macro-micro-macro. Los modelos de redes representan, de manera más sutil que los modelos multinivel, que lo que puede ser observado a nivel macro es el resultado de fenómenos a nivel micro, mientras que estos fenómenos están condicionados por el nivel macro. El uso de modelos estadísticos apropiados para redes, con atención suficiente al ajuste del modelo, puede conducir a progresos importantes en nuestra comprensión de las cuestiones macro-micro-macro. Sin embargo, esto es más una promesa de cara al futuro que un logro del pasado. Una razón de importancia mayor de las limitaciones en el estado actual del arte es que las cuestiones de retroalimentación (feedback) que son esenciales en cómo operan 
las redes, son extremadamente difíciles de expresar en modelos estadísticos de forma operativa y plausible.

En los modelos para una única observación (en un momento dado) de una red social, la dimensión temporal de la retroalimentación es aplastada, lo que conduce a dificultades graves tanto de cara a la interpretación como al modelado matemático. A pesar de que modelar observaciones en un momento dado de las redes sociales es todavía necesario y útil, es posible ganar en comprensión a partir de observaciones repetidas de redes sociales (en varios momentos diferentes). Si tal modelo dinámico incluyese, no sólo las relaciones entre actores, pero también los atributos cambiantes de los actores (p.e., las actitudes, comportamientos, resultados) y las pautas englobantes de los contextos sociales en que las redes se encuentran inmersas, capturaría una gran parte de lo que consideramos como procesos macro-micro-macro. Tal modelo integrado no existe todavía, pero se están llevando a cabo avances hacia la construcción de este tipo de modelo.

\section{Posiciones en las redes y modelos de grafos aleatorios}

Philippa Pattison y Garry Robins

El objetivo de este artículo es yuxtaponer dos aproximaciones aparentemente diferentes al modelado de la estructura de las redes: las aproximaciones posicionales como el blockmodeling que presuponen una clasificación de los nodos de una red en posiciones heterogéneas en las redes y los modelos de grafos aleatorios que están basados en la proposición de procesos locales homogéneos. Analizamos las bases teóricas de estas dos perspectivas y utilizamos simulación así como el estudio de un caso para explicar las similitudes y diferencias entre ellos. Concluimos con un número de recomendaciones para orientar las investigaciones empíricas y teóricas futuras.

\section{Dualidad y la agregación de categorías sociales}

Ronald Breiger

La "dualidad" una manifestación distintiva de la relación micro-macro se refiere a la co-constitución de elementos existentes a diferentes niveles de la estructura social. Por ejemplo, los grupos se distinguen por las redes de miembros que los constituyen, igualmente que las personas pueden ser identificadas (siguiendo a Cooley, Simmel y Goffman) mediante las redes o grupos a los que pertenecen. Teóricos sociales tan distintos como Coleman y Bourdieu han utilizado nociones de dualidad al estudiar las relaciones micro-macro. Existe un nuevo énfasis (como en los trabajos de Mohr y Duquenne) sobre la dualidad de las prácticas locales y los símbolos culturales globales y los roles.

Tras explicar la dualidad como un tipo distintivo de relación micro-macro, presento un algoritmo útil para implementar la agregación dual de las categorías de la fila y la columna de una clasificación cruzada, incluyendo tablas en que las filas y las columnas dan cuenta de diferentes tipos de entidades (como grupos culturales y prácticas locales) y tablas uni-modales (como las tablas de movilidad). Además, los conceptos de equivalencia de red se han mostrado útiles en el modelado de la agregación de categorías sociales en las tablas de clasificaciones cruzadas. Se enfatiza la aplicación al análisis de tablas de contingencia de la dualidad de la cultura y las prácticas.

\section{Una nueva perspectiva sobre la equivalencia estructural: lugares y redes de lugares como instrumentos para la teoría sociológica} Narciso Pizarro

La definición del concepto de lugar como un instrumento para describir redes duales. Las redes de lugares como redes reducidas: los lugares son clases de individuos estructuralmente equivalentes. El álgebra de las redes de lugares. Relaciones directas e indirectas en las redes de lugares. La dualidad de las redes de 
lugares. El álgebra de las redes de lugares como un caso particular del tipo de bandas rectangulares: el semigrupo de banda cuadrada. Redes de lugares y estructura social.

\section{Una perspectiva institucional sobre la relación micro-macro}

Wouter de Nooy

La relación micro-macro puede ser comprendida como un proceso dinámico en el que los actores interpretan los modelos locales de relación en tanto que indicadores - elementos de una estructura de conjunto, comunicar sus interpretaciones, y ajustar sus relaciones hacia la estructura en su conjunto tal como la perciben. En este artículo, se propone que los actores perciben las subestructuras locales en una red de evaluaciones, como las díadas, tríadas o semiciclos cortos, e infieren agrupamiento y jerarquización de forma que son compatibles con modelos de teoría del equilibrio (consistencia cognitiva?). Interpretan y comunican la información como clasificaciones simplificadas e idealizadas parecidas a los blockmodels respecto a los que ajustan sus relaciones después. De este modo, las perspectivas ego-centradas y socio-centradas se relacionan de manera dinámica.

Esta perspectiva es aplicada a evaluaciones entre autores y críticos in las instituciones literarias. En el nivel micro, los autores literarios y los críticos ajustan sus evaluaciones a las evaluaciones precedentes. En el nivel global, la institución literaria es estratificada en grupos, por ejemplo movimientos literarios y estilos. Los miembros de esta institución se reflejan en su estructura: clasificaciones de acuerdo con el movimiento y el estilo son comunicadas y discutidas en la crítica literaria. 